\title{
Kernos
}

Revue internationale et pluridisciplinaire de religion grecque antique

$4 \mid 1991$

Varia

\section{Le Dionysos oraculaire}

\section{Ileana Chirassi Colombo}

URL : http://journals.openedition.org/kernos/301

DOI : 10.4000/kernos.301

ISSN : 2034-7871

\section{Éditeur}

Centre international d'étude de la religion grecque antique

\section{Édition imprimée}

Date de publication : 1 janvier 1991

Pagination : 205-217

ISSN : 0776-3824

Référence électronique

Ileana Chirassi Colombo, «Le Dionysos oraculaire », Kernos [En ligne], 4 | 1991, mis en ligne le 11 mars 2011, consulté le 20 avril 2019. URL : http://journals.openedition.org/kernos/301 ; DOI : 10.4000/ kernos.301 
Kernos, 4 (1991), p. 205-217.

\section{LE DIONYSOS ORACULAIRE}

Pour l'homme grec de l'âge archaïque et classique, et bien avant encore, l'objet épistémologique par excellence est un savoir global qui, d'Homère à Plutarque, peut être défini comme un art divinatoire qui s'exerce sur "ce qui est, ce qui sera et ce qui fut» ${ }^{1}$. C'est un domaine qui, comme dit Ammonios dans un passage du De E apud Delphos de Plutarque, est en particulier le domaine des dieux, ou mieux le domaine d'un dieu philosophos, qui se plaît au savoir et qui le possède en propre ${ }^{2}$. Ce dieu est Apollon, bien sûr, le maître de Delphes, assimilé au soleil qui peut tout voir, tout savoir et tout communiquer par différents moyens à qui il veut. C'est le dieu mantique par excellence, Apollon, fils de Zeus. Mais n'oublions pas que c'est précisément au cœur du domaine mantique, à Delphes, qu'un autre fils de Zeus, Dionysos, n'a pas une part moindre qu'Apollon ${ }^{3}$. L'équivalence du pouvoir entre Apollon et Dionysos, et le partage du territoire commun est envisagé ici d'un point de vue théologique qui fait apparaître Dionysos comme le double polymorphe et pathétique, toujours en transformation, de l'Un Apollon, égal à soi-même et immobile. Mais, à bien regarder, cette équivalence, antithétique en apparence seulement, se fonde sur leur participation commune à la même fonction, à savoir la réglementation du territoire très ambigu de l'atechnos mantikè, c'est-à-dire la mantique inspirée, ou encore ce savoir que l'on peut obtenir par intervention directe de la divinité, ou par des capacités extraordinaires d'ordre naturel. Un naturel qui en tout cas peut être bien "divin" en soi si, ouvertement, dans la perspective stoïcienne au moins, on peut considérer la physis, la nature, comme l'essence originelle de la divinité coïncidant avec la loi du kosmos.

Dans le monde grec des poleis, l'atechnos mantikè occupe une place à part qui contraste avec la grande variété des possibilités mises en œuvre par la mantique technique, qui est avant tout l'art de Calchas, le premier mantis de la tradition grecque, et le seul type de divination

1 C'est le savoir de Calchas : Ном., Il., I, 70.

2 PLUT., Sur l'E de Delphes, 387b-c.

3 Macrobe, Sat., I, 18; cf. aussi P. Amandry, La mantique apollinienne à Delphes, Paris, 1950, p. 196 sq. 
apparemment connu d'Homère ${ }^{4}$. La différence entre les deux types n'est pas seulement une question de procédure, mais aussi et surtout de fonction au niveau politique et idéologique. Le devin qui exerce la mantique technique travaille comme un interprète de signes, chargé de comprendre les messages cachés sous la pluralité de signes éparpillés dans le cosmos visible et par lesquels, comme l'affirmait l'école stoïcienne, les dieux révèlent aux hommes le grand plan du monde.

C'est dire que, dans une perspective "religieuse», la divination en vient à être une preuve de l'existence même des dieux qui, selon une vetus opinio omnium gentium firmata consensu, selon une koinè ennoia de l'humanité, non seulement existent, mais connaissent l'avenir et peuvent le révéler, semainein, par différents moyens ${ }^{5}$. Une autre interprétation, que l'on peut qualifier de «laïque», confirme la même possibilité en soulignant l'existence d'un plan, d'un programme, d'un logos du monde qui fonctionne comme un système d'écriture et permet au technicien alphabétisé de lire les res quae dicuntur en utilisant les prodiges, les semeia, comme les vocabula quibus res dicuntur. Mais la mantique qui nous concerne, la mantique dionyso-apollinienne, est une affaire toute différente. Le plan du monde peut être déjà établi ou bien encore en train d'être tissé, mais, quoi qu'il en soit, les puissances extrahumaines, dieux ou démons, ou les puissances agissant à l'intérieur des hommes, ou mieux la physiologie très particulière de certains individus, peuvent dévoiler l'avenir et le communiquer à tous par le moyen commun du langage humain. À ce moment, le langage devient très important parce que ses amphibologies, ses métaphores, sa faiblesse dans la construction du rapport entre la chose et le signe, la parole, signifient l'ambiguïté de la communication, l'entrave qui empêche la circulation du message entre destinateur et destinataire. L'examen de ce processus, quoique important, n'entre pas dans le champ d'intérêt qui nous concerne ici.

C'est la modalité de la transmission qui nous retiendra. Cette modalité fait la différence entre le genos technikon et l'adidaktos kai atechnos mantikè, ou bien entre le genus divinandi artificiosum et le

4 Cf. Ileana Chirassi Colombo, Gli interventi mantici in Omero, morfologia e funzione della divinazione come modalità di organizzazione del prestigio e del consenso nella cultura greca arcaica e classica, in F.M. FALES - C. GROTTANELLI (ed.), Soprannaturale e Potere nel mondo antico e nelle società tradizionali, Milano, 1985, p. 161-164.

5 Cic., Div., I, 10; 25; 92; II, 80; cf, aussi Lois, II, 32-33. Pour le Stoïcien ChrYsIPPE, la divination est la faculté de connaître, de voir, d'expliquer les signes offerts aux hommes par les dieux (Crc., Div., II, 80). 
genus naturale, entre ars et natura, comme dit Cicéron, où l'on peut noter d'abord l'aporie ou mieux l'impossibilité de traduire atechnos par naturale 6 . Ainsi le même Cicéron introduit pour précision le trio deus, fatum, natura : duxisti enim divinationem omnem a tribus rebus, a deo, a fato, a natura. Au niveau de la définition de fatum et du terme grec correspondant, la discussion pourrait traîner en longueur. Une mise au point sur le rapport entre fatum, destin inéluctable, et divination est présentée par Cicéron lui-même dans un traité particulier, le De Fato, qui nous est parvenu très mutilé mais qui nous permet en tout cas de connaître les arguments de l'Académie (Carnéade) contre le déterminisme stoïcien, garant par excellence de la mantique technique. On garde ainsi l'élément très important de la possibilité d'intervention des dieux (et en conséquence des hommes) qui peuvent véritablement bouleverser toutes les prévisions par leur volonté 7 .

L'objet de notre étude sera la qualité extraordinaire de la communication définie par Cicéron $a$ deo et a natura. Dans ce cas, la révélation de l'avenir est réservée à l'intervention directe des dieux, ou bien elle dépend d'un savoir qui est lié à une qualité intérieure de l'individu, quelque chose qui fait partie de sa physiologie et dès lors la personne se trouve différente des autres, en dehors de l'humanité ${ }^{8}$. L'importance de

6 CIC., Div., I, 12; Ps.-Plut., Homère, 212. Atechnos indique simplement que le divin n'utilise pas un instrument, une clef de lecture, mais qu'il entre par différents moyens dans une dimension de connaissance supérieure qui peut ou non dépendre de l'intervention d'une divinité. ARISTOTE s'interroge sur certains individus tout à fait simples qui sont capables de donner des prévisions exactes dans leurs songes; dans ce cas, «ce n'est pas un dieu qui envoie ces révélations, mais chez tous ceux dont la nature se présente comme si elle était bavarde et mélancolique, on trouve des visions variées" : ARISTOTE, De la divination dans le sommeil, $463 \mathrm{~b}$.

7 CIC., Div., I, 125. Le fatum traduit le grec heimarmenè la fatalis necessitas, qui permet de lire le monde. Mais la dimension prophétique comme communication de la part de dieux admet aussi la possibilité du changement. Voir le commentaire de Servius : Si vox lovis fatum est, potest aliud fando fati ordinem commutare (ad Ain., X, 628). Ainsi interprété, le fatum devient la force de la communication directe, la valeur inépuisable de la parole. Apollon peut prévoir des événements qui n'ont dans la nature aucune cause, seulement si ces événements ont pour cause sa volonté ou la volonté de Zeus (CARNÉADE dans CrC., Du destin, 33-35). Cf. F. GuILlaumonT, Philosophe et augure. Recherches sur la thérie cicéronienne de la divination, Bruxelles, 1984, p. 159 sq.

8 C'est la melancholikè krasis, ce mélange particulier de la bile noire que produisent les individus d'exception, comme les héros du temps jadis, mais aussi des personnages illustres plus récents, philosophes, chefs militaires et ... devins, comme les Sibylles, les Bacis et tous ceux qui sont inspirés (entheoi): 
la personne à laquelle est reconnu un pouvoir de connaissance "divine», naturelle, c'est-à-dire inclu dans sa constitution physique, est bien claire et, dans la perspective du prestige, égale au charisme du choix divin qui rend la mantique inspirée particulièrement importante. Pour cela, il suffit de citer Platon dans le Timée quand il fait la distinction entre les prophètes qui sont interprètes des paroles ou des signes envoyés par les dieux, et les véritables manteis qui transmettent directement la parole, la volonté des dieux (Timée, 71-72b).

Après Platon, il faut encore rappeler Plutarque quand, dans le $D e$ Genio Socratis, il sépare bien les sacerdoces techniciens, les manteis qui s'occupent des oiseaux, des autres, les quelques privilégiés qui ont la chance de rencontrer le dieu : parce que, dit-il, «le dieu ne rencontre pas beaucoup de monde, mais il donne à beaucoup ses signes» (593c-d). La communication directe divin-humain ou la présence dans le corps humain d'une qualité, d'un mélange qui exprime une puissance que l'on peut qualifier de divine, prend la signification d'une investiture charismatique qui isole l'individu ainsi doué dans une situation marginale très signifiante.

La reconnaissance culturelle d'une situation de ce type peut devenir un instrument efficace pour déterminer le comportement individuel et collectif des membres du groupe, influencer leurs choix. Comme on l'a déjà observé, la Grèce des cités, la Grèce archaïque et classique, ne connaît pas, ou mieux ne veut pas connaître l'exercice de la mantique inspirée par un corpus de «spécialistes" comme les neb̂̂îm d'Israël ou les prophètes de Mari ${ }^{9}$. C'est-à-dire que la Grèce ne veut pas produire des personnages qui agissent individuellement dans la société comme porteurs d'un message qui concerne le comportement collectif et le programme futur, et qui doit être reconnu comme vrai. Le monde des cités relègue la foule des Sibylles, les prophètes femmes, dans

ARISTOte, Problème XXX, 1, 953a 10. Cf. J. PigEAUd, L'Homme de génie et la mélancolie, Paris, 1988. Pas de surprise dans l'absence de la Pythie : elle n'a pas une physis exceptionnelle, mais elle est seulement l'instrument du dieu. Comme la nymphe Syrinx qui métamorphose son corps en roseau pour offrir enfin à son persécuteur, le dieu bouc Pan, spécialiste de la possession, un instrument musical nouveau. Cf. Ph. Borgeaud, Recherches sur le dieu Pan, Roma, 1979 (Biblioteca Helvetica Romana, 17), p. 124 sq.

9 La divination inspirée, par la possession d'une divinité, est attestée en Syrie, bien avant l'expérience du prophétisme hébreu. Cf. la documentation très riche dans le Dictionnaire de la Bible, Suppl. VIII, Paris, 1972, s.v. prophétisme. Pour Mari, il faut signaler la grande diffusion du porphétisme féminin : $\mathrm{cf}$. G. Dossin-A. Finet, Archives Royales de Mari. Correspondance féminine, Paris, 1978. 
l'imaginaire clos du temps mythique, dans le monde non encore apollinisé ${ }^{10}$. La cité iso- ou eunomique qui, comme dit Aristote, poursuit son utopie, l'égalité, avant toute autre chose (Politique, 1284), est obligée de refuser les savoirs qu'on ne peut pas contrôler. Pas seulement les savoirs mantiques, bien sûr, mais tous les types d'un savoir excessif et inutile : le savoir du sophos Anaxagore ou bien de la magicienne Médée, ou de la Sibylle. Ainsi, pour contrôler un savoir utile et en même temps dangereux, supposé venir $e k$ theou, de dieu, le savoir qui «doit» être vrai et bon, que Platon oppose au savoir de la science humaine soumis aux lois du nous et de l'historiè, exposé aux risques de l'oiesis, l'opinion, les cités inventent un "métacentre» où rassembler tout ce qui se produit par la volonté du dieu. On peut aisément découvrir cette idéologie en filigrane du plan de fondation de Delphes comme nombril du monde et comme lieu privilégié de la communication entre homme et dieu, un type de communication qui doit être limité.

Ainsi un corps vide de femme se fixe comme le moyen visible et permanent d'une circulation du logos strictement contrôlée qui permet le déroulement d'une histoire préalablement décidée dans ses lignes fondamentales. C'est bien la Pythie dans son rôle de prophète, de portevoix d'Apollon, d'organe du dieu, instrument docile qui «se conforme le mieux possible à l'agent qui l'emploie» (Plutarque, Des oracles de la Pythie, $404 \mathrm{~b})^{11}$. Dans cette perspective il faut reconnaitre que la Pythie réalise en elle au maximum les potentialités implicites du processus de la transe de possession, qui est la modalité préférée de la communication entre l'humain et l'extra-humain réalisée par la transe rituelle très longuement étudiée par les anthropologues, les historiens des religions et dans les domaines de la psychiatrie et de la psychanalyse ${ }^{12}$.

10 Cf. le «type sibyllin» dans l'ouvrage, ancien mais non remplacé, de A. BOUCHÉLECLERCQ, Histoire de la divination dans l'antiquité, II, Paris, 1879 [1963], p. 133 sq.

11 Sur la qualité de l'inspiration de la Pythie, voir AMANDry, op. cit., p. 19 sq et la discussion chez A. PINERO SAENZ, Sobre la inspiration de la Pitia delfica. Breve historia de una polemica, in Durius, 3 (1975), p. 405-416. Mais la question est en général mal posée. Voir infra.

12 Cf. G. Rouget, La musique et la transe, Paris, 1980, p. 58 sq. : pour l'auteur, la Pythie constitue l'exemple illustre d'un type particulier de transe médiumnique dans laquelle le personnage inspiré ne s'identifie pas à son dieu mais délivre seulement sa parole. Même procédure dans le prophétisme syrien et hébreu. Erica Bourguignon (Psychological Anthropology. An Introduction to Human Nature and Cultural Differences, New York, 1979) fait une distinction entre la transe d'expérience, ou «chamanique», dans laquelle l'individu vient au contact 
Le lexique grec utilise pour définir le statut de la possession une terminologie très variée. Le terme le plus explicite est certainement le vocable entheos, dans le sens d'être investi par le dieu, "endieué»13. Ce lexique de la possession est bien établi dans l'usage grec du Ve siècle av. J.-C. Dans les Sept contre Thèbes, entheos Arèi, "endieué" par Arès, est le guerrier Hippomédon qui, en conséquence, bakhai, fait le bacchant comme une thyias, une Thyade (497-499). Ou le parallèle qui s'impose peut-être avec la rûăh yhwh, le souffle, l'esprit de Dieu qui vient sur Saul, l'enflamme de rage et le pousse à la victoire sur les Ammonites (I Samuel, XI, 6). Dans l'Antigone de Sophocle, entheoi gynaikes sont les femmes du cortège du Dionysos, les Ménades (962). Pour elles, le dieu dedans, le dieu qui les occupe est certainement le mainomenos par excellence, le fou, Dionysos. Mais Dionysos n'est pas le seul dieu de la possession comme il n'est pas non plus le bakhos originel, d'autant que bakhos ne semble pas désigner une divinité personnelle, mais plutôt une modalité du comportement et de l'être, une expérience. Le lexique grec qui se concentre autour de cette expérience est riche et ambigu en même temps, et nous n'avons pas ici la possibilité de passer en revue la problématique complète qui le concerne. Entheos intrigue avec bakhos dans la construction du même message avec un grand nombre des composés de entheatho, comme enthousiastikos, entheastikos, etc., qui désignent la situation d'un être pris par une entité, une divinité $e k$ tinos. Quelque chose ou quelqu'un qui occupe, qui tient, katechein, et fait d'un individu un katochos. Les poètes sont katechomenoi hosper hai bakhai, selon le lexique de Platon (Ion, 532), comme les individus qui se font "corybantiser" dans le rituel complexe lié à la collectivité des Corybantes ${ }^{14}$. C'est une situation qui, très facilement, peut avoir une solution du type médianique dans laquelle le possédé, dans un état de dissociation complète ou bien partielle, vient à

de différentes puissances et différentes situations qu'il peut évaluer et utiliser indépendamment, et une transe de possession où l'individu est envahi par une puissance externe avec laquelle il s'identifie au point de perdre conscience de soi. Il est intéressant de noter que la transe chamanique est «masculine» alors que la possession est "féminine»!

13 Cf. Rouget, op. cit., p. 273. Amandry traduit l'entheos de la Pythie comme un "état de grâce" sur la suggestion inconsciente (!) des métaphores de l'annonciation dans les évangiles. Cf. E. Norden, Geburt des Kindes. Geschichte einer religiösen Idee, Stuttgart, 1969 [1924], p. 87 sq.

14 Cf. P. BoyancÉ, Le culte des Muses chez les philosophes grecs, Paris, 1972 [1936]. I. LINForTh, Coribantic Rites in Plato, UCP in Cl.Phil., 13 (1946), p. 121 162; RouGET, op. cit., p. 267 sq. 
transmettre un savoir qui peut parvenir par voie hypnotique mais qui peut être reçu comme la parole, le logos, verbum d'un dieu ou du dieu ${ }^{15}$. Le statut de la possession médianique où l'on peut se manifester par un message non verbal, visuel, celui de l'altération physionomique, de la gesticulation, du mouvement rapide du corps qui envoie l'avertissement de la nouveauté de la situation dans laquelle la personne se trouve plongée. C'est le cas typique de la bacchante. Mais on peut trouver aussi un type de possession qui ne présente aucune manifestation spectaculaire et dont les symptômes sont presque imperceptibles.

Ce type d'expérience, dans toutes ses manifestations, a été décrit dans la culture grecque, en particulier, mais pas uniquement, dans le lexique tragique, comme la manifestation par excellence d'un contact avec un dieu ou bien comme manifestation du statut très ambigu de la mania. Au Ve siècle, la possession par intervention directe des dieux semble très répandue. Un passage bien connu de l'Hippolyte d'Euripide nous donne une liste : Phèdre, amoureuse d'Hippolyte, ressemble à un possédé de Pan, d'Hécate, ou à un égaré par les saints Corybantes ou par la Mère des Montagnes (141). Le texte est significatif parce que le comportement de Phèdre n'a rien de violent, elle semble seulement rêver à une fuite dans la montagne, à une participation à l'engagement masculin de la chasse (214-226), à l'agitation du statut de possédé. Elle voudrait bien être Artémis, la déesse aimée par Hippolyte ! En réalité, elle demeure dans une tristesse qui la tient enfermée dans sa chambre (161 sq.).

Presque toutes les divinités à partir du Ve siècle se spécialisent dans la pratique de la possession, ce type très privilégié de communication avec l'humain. On trouve une autre liste dans le traité pseudo-hippocratique Sur la maladie sacrée. On peut ajouter la collectivité des nymphes responsables de la nympholepsie, une forme très particulière de capture de la personne par une véritable inondation de substance divine ${ }^{16}$.

15 Cf. Giulia SISSA, La Pizia delfica : immagini di una mantica amorosa e balsamica, in Aut Aut, 184-185 (1981), p. 193-213.

16 Nympholeptos, possédé par les nymphes, est Socrate dans le lieu extra-urbain qui vient d'être le cadre du discours sur l'amour de l'âme (Platon, Phèdre, 238c). Un autre est le chresmologue béotien Bakis, «fou par les nymphes» (PAUS., IV , 27, 4), personnage historique incertain qui aurait donné des prévisions valables pendant les guerres médiques (HDT., VIII, 20, 77; IX, 43). Au Ve siècle, la nympholepsie se présente au niveau historique comme une expérience assurée par les nymphes de la grotte de Pan à Vari, en Attique. Voir les dédicaces votives d'Archédémos de Théra (I.G., II², 788). L'essor mantique de ce type de possession est assuré non seulement par la tradition grecque qui 
Nympholeptos, pris par les nymphes, précise theoleptos, pris par un dieu quelconque, tandis que phoiboleptos, désigne l'emprise d'Apollon qui peut entraîner l'expérience structurellement différente de l'extase, par laquelle l'âme et le corps se séparent ${ }^{17}$. On connaît l'exemple d'Aristée du Proconnèse qui voyage avec Apollon sous la forme d'un corbeau (Hérodote, IV, 15). La description peut-être la plus claire d'un statut de possession est donnée par un philosophe, en l'occurrence Platon, dans le Phèdre. Le comportement de l'âme qui se rapproche de son dieu est pareil au comportement des enthousiontes ek theou tinos, de ceux qui sont possédés par un dieu de façon à lui emprunter leurs comportements et leur activité, pour autant qu'il soit possible à l'homme de participer à

connaît dans une autre grotte, un manteion administré par des nymphes, le Sphragidion du Cithéron (PAUS., IX, 3), mais indirectement par la tradition latine. Traduit par lymphatus (VARRON, Lang. lat., VII, 87; Cic., Div., I, 80), le mot indique la modalité "hydrique» de la procédure oraculaire. L'empire de la nymphe est la pénétration ambiguë d'un flux que le fragment tragique de PACUVIUS, cité par VARRON, met en rapport avec l'excitation bachique : flexanima tamquam lymphata aut Bacchi sacris/commota. Lymphata dicta a lympha; <lympha> a Nympha in Graecia commota mente quos nympholeptous appellant et ab eo lymphatos dixerunt nostri. "Les nymphes versaient dans l'âme humaine un trouble surnaturel qui revêtait toutes les formes", écrivait BOUCHÉ-LECLERCQ (op. cit., p. 263). Le flux des nymphes s'apparente ainsi aux différents pneumata qui rendent l'âme féconde. La forme «phallique» de la nymphe comme femme serpent ou poisson, sirène ou fée comme la médiévale Mélusine, traduit la force de la métaphore qui privilégie, dans la description de la possession comme acte sexuel, le rôle toujours «actif» donné au mâle. Cf. I. ChIRAssi-Colombo, Melusina o il segno del serpente : avventure stori co-culturali di una donna anche serpente, in Melusina. Mito e leggenda di una donna serpente, Roma, 1986, p. 61-104. Pour le rapport entre possession et divination, voir E. BouRGUIGNON, Divination, transe et possession en Afrique transsaharienne, in La Divination, études recueillies par A. CAQUOT et M. LEIHOVICI, Paris, 1968, p. 331-358.

17 Ekstasis indique l'action de se déplacer, ou mieux d'être hors de soi, ou l'aventure d'une exploration de l'âme au-dedans de soi par un chemin intérieur selon l'enseignement de PlotiN (Ennéades, V, I, 10). Dans la tradition grecque le premier aspect, le plus clairement "chamanique» est bien attesté par Hermotime de Clazomènes qui quittait souvent son corps pour faire ses voyages en tant qu'âme (PLINE, Hist. Nat., VII, 174) tandis que les aventures de personnages comme Abaris, Aristée, même Pythagore proposent le phénomène différent de la translation bien connu dans la tradition juive et chrétienne. Cf. I. Culianu, Expérience de l'extase, Paris, 1984, p. 22-43; I. Chirassi Colombo, Misticismo, mistica, estasi : il lessico storico nell'occidente precristiano, in Mistica e Linguaggio, Trieste, 1986, p. 7-34. La possession implique au contraire l'occupation d'un vide humain, l'individu dissocié, par une puissance extérieure. Aspects et résultats sont opposés, cf. n. 12. 
la divinité (253a). La modalité de la participation envisagée comme une occupation du vide humain par la substance divine peut se résoudre dans la mise en scène d'une véritable pièce de théâtre où les dieux semblent jouer leur épiphanie parmi les hommes en devenant pour quelque temps des humains. Quelquefois, la possession peut aboutir à une modalité de communication très particulière, à la mise en action d'un programme oraculaire, dans lequel, comme le dit encore Platon dans l'Ion, c'est la divinité elle-même qui parle par l'intermédiaire du corps humain.

La divinité leur ôte la raison et, en les prenant pour ministres comme chresmôidoi et manteis divins, fait apprendre à nous les auditeurs que ce n'est pas eux, les manteis, qui disent les choses mais la divinité elle-même qui parle et, par leur intermédiaire, se fait entendre à nous.

(Platon, Ion, 534b)

L'organisation métaphorique du langage prête à la communication oraculaire par possession plusieurs modèles. Le plus connu, comme nous l'avons déjà noté à propos des nymphes, organise le processus de transmission du savoir comme la pénétration d'un liquide, d'un souffle, d'un rayon, qui peut "métonymiser» le dieu lui-même, ou mieux sa puissance. C'est ainsi que la mantique apollinienne est en général liée à l'eau ou à la lumière tandis que la mantique dionysiaque est liée au vin. Toutes deux peuvent être également pneumatiques. La symétrie eau/vin comme analogie de la symétrie - par implication et non par opposition - Apollon/Dionysos dans le cadre de la communication oraculaire est très bien illustrée dans l'exemple choisi par Macrobe. Il s'agit d'un passage des Saturnales consacré à l'identification Apollon-Dionysos proposée par la théologie delphique : Apollinem et Liberun patrem unum eundumque deum esse (I, 18). Parmi les arguments présentés, on cite expressément la participation conjointe des deux dieux au domaine du savoir oraculaire inspiré. En Thrace, il y a un adyton oraculaire consacré à Liber-Dionysos où la procédure de consultation prévoit pour les váticinaturi, les prophètes, la consommation de merum, vin pur, non mélangé, exactement comme dans le Clarion, le fameux sanctuaire d'Apollon près de Colophon où l'on peut vaticiner en buvant de l'eau. L'eau est présente dans beaucoup d'endroits de la mantique apollinienne en Asie Mineure, à Colophon comme à Milet, mais aussi à Delphes où les jeunes filles qui deviennent hiérodules, esclaves du temple, prennent un bain purificateur dans l'eau prophétique de la source Kastalie et, comme le précise la scholie, 
s'apprêtent à prophétiser ${ }^{18}$. L'eau apollinienne renverse le signe du vin qui manifeste Dionysos dans la proposition d'un statut "pneumatique» commun supposé par la fluidité qui permet l'accès à la sobria ebrietas, l'ivresse sans vin. Une situation que nous pouvons décrire avec les mots employés par Philon pour évoquer l'expérience du dialogue avec Dieu pratiquée par la communauté, peut-être utopique, des Thérapeutai d'Égypte, ou pour commenter l'ivresse mystique d'Anne, mère-prophète et mère du prophète Samuel, prophètis kai prophètotokos (Philon d'Alexandrie, Des songes, 254; De l'ivresse, 95; 128; passim) ${ }^{19}$. Avec le vin et l'eau, Dionysos et Apollon se rapprochent sous le signe du flux, du reuma générateur du pneuma, esprit, écume bouillonnante, matière non matière, générateur de chaleur, fécond en soi, analogue au sang, au sperme, à la substance divine. Tous les souffles viennent de l'eau, ta de pneumata hèmin esti panta aph'hydatos, dit l'auteur du traité Sur la nature de l'enfant du Corpus Hippocratique (25 Littré). Dans un passage fort intéressant, Plutarque cherche à expliquer par une interprétation partiellement physique la divination inspirée, et parle d'un mantikon reuma kai pneuma, flux et souffle divinatoire qui est en soi theiotaton, très divin (Plutarque, Sur la disparition des oracles, 432d).

La dimension pneumatique unit Apollon et Dionysos dans le privilège de faire participer les humains au pouvoir créateur, et donc divin, qui réside dans la qualité du pneuma, la substance qui, grâce à sa qualité analogue au sperme mâle, renferme en soi la force créatrice qui distingue aussi, à partir de la spéculation d'Aristote, le caractère spécifique de la divinité post-polythéiste ${ }^{20}$. Ainsi la notion de pneuma, centrale pour l'organisation de la vision cosmique grecque, grâce au jeu inépuisable des chaînes métaphoriques, permet de dire l'imaginaire théologique dans une terminologie très variée. La qualité cinétique du pneuma traduit en effet l'essence, l'ousia, de la divinité, et la manifeste dans la créativité au sens large. Le poète, le dèmiourgos, créateur par

18 Eur., Phén., 222-225 et Schol.ad 1. La Pythie est plutôt en rapport avec la source Cassotis (PAUS., X, 24, 7). Sur l'eau et les travaux de canalisation dans les sanctuaires d'Apollon, cf. R. GINouvès, Balaneutikè. Recherches sur le bain dans l'antiquité grecque, Paris, 1962, p. 235 sq.

19 Philon D'AleXANDrie, De la vie contemplative. H. LeWy, Sobria Ebrietas. Untersuchungen zur Geschichte der antiken Mystik, Giessen, 1929.

20 Cf. H. LeISEgaNG, Der heilige Geist. Das Wesen und werden der mystichintuitiven Erkentniss in der Philosophie und Religion der Griechen, LeipzigBerlin, 1919. Cf. G. VERBEKe, L'évolution de la doctrine du pneuma. Du stö̈cisme à S. Augustin, Paris, 1945; G. KitTeu (ed.), Theologisches Wörterbuch zum Neuen Testament, Stuttgart, 1959, s.v. pneuma (H. Kleinchnet). 
excellence de la société grecque archaïque, peut créer seulement dans l'enthousiasmos, quand un hieron pneuma, un souffle saint, le pénètre (Démocrite, cité par Clément d'Alexandrie, Stromates, VI, 168). Mais la métaphore va plus loin. Ainsi le souffle de Zeus, ek epipnoias Zènos, provoque la conception d'Épaphos; le fils naît d'une caresse du dieu à Io, la génisse argienne persécutée par la jalousie d'Héra, dans les Suppliantes d'Eschyle. Et c'est encore un souffle "du dieu", un atmon entheon, qui provoque la grossesse de la Pythie, une grossesse de paroles, de discours, du logos divin, pour l'auteur du Traité du Sublime (XIII, 2). L'image traduit très bien la modalité avec laquelle Apollon s'approche de la femme qui lui produira son discours. Il s'agit d'un type de possession moyenne dans laquelle il n'y a pas d'identification avec l'être surnaturel, mais un échange conçu en termes - bien connus des langages mystiques - de rapport sexuel, qui peut être raconté selon plusieurs variantes.

Dans ce cas-là, il s'agit d'un souffle tellurique pareil aux souffles telluriques bien connus en différents endroits de la terre. Mais ce souffle peut être une propriété du corps, ou un cadeau de la terre, ou l'essence même de la divinité.

Comme porteur du mouvement, comme heauton kinoun, le pneuma exprime l'autokinèton athanaton, l'automoteur immortel, l'âme et le dieu pour Platon (Phèdre, 245c), ou bien le pouvoir créateur du sperme mâle qui seul, dans la théorie physiologique d'Aristote, est le principe de la génération qui réside dans le mouvement et la chaleur produite par le mouvement (Aristote, De la génération des animaux, 736a-737a). En conséquence, le pneuma est essentiellement fécond comme le dieu mâle, le dieu universel que les Stoïciens identifient au logos spermatikos, le discours, le projet séminal, qui dans l'air et dans l'humide assure l'existence du monde (Diogène Laërce, VII, 136). C'est le principe qui peut s'identifier à Zeus ou à un dieu quelconque quand, en s'approchant d'une femme, il produit en elle les conditions nécessaires à la naissance ${ }^{21}$. La puissance pneumatique assure en tous cas la réalisation d'un message, la délivrance d'un signe qui provoque la production de la communication. Dionysos et Apollon se coalisent dans ce projet. Le chœur des Bacchantes d'Euripide chante Dionysos comme le daimon qui, par la fureur qu'il inspire, transmet un pouvoir prophétique pénétrant tous les êtres de sa puissance en poussant à dire l'avenir (298 sq.). Comme la rûăḥ'ělohîm provoque la parole dans la bouche de son porte-parole (2 Samuel, 23, 2). Et Apollon produit dans le corps choisi de

21 Plut., Numa, 4; Is. et Os., 36. 
sa femme prophète le principe du mouvement, l'archè tès kinèseôs, provoquant en son âme la lumière qui éclaire l'avenir, le phôs pros to mellon $^{22}$. Cette qualité lumineuse du pneuma est bien attestée dans la tradition et ne contredit aucune de ses possibilités. Pour les Thérapeutai de Philon d'Alexandrie, «l'âme aimée de Dieu engendre en vertu de la semence paternelle qui est un rayonnement de l'intelligible" (Vie contemplative, 68). La prophètis d'Apollon à Didymes, illuminée par son dieu, peut devenir pleine de sa splendeur divine et parler (Jamblique, Les Mystères d'Égypte, III, 11) ${ }^{23}$. Mais on peut convenir que la possession dionysiaque ne produit pas le même effet que la possession par Apollon. Du moins en apparence, bien que l'on ait dit que la pénétration du pneuma dans l'âme produisait les effets des vapeurs du vin (Plutarque, De la disparition des oracles, 432e). La possession d'Apollon poursuit un but précis : un logos, un discours sonore, ... un fils. La Pythie devient régulièrement grosse des paroles légitimes, à l'inverse de Cassandre qui, par sa "faute», reste stérile. La symétrie est évidente. Dans l'Agamemnon d'Eschyle, Cassandre explique au chœur comment Apollon lui avait donné la faculté de la prophétie. La modalité du rapprochement du dieu et de la fille est immédiatement comprise par le chœur en termes de poursuite sexuelle. Cassandre ne voulait pas parler, par aidôs, par pudeur, mais elle parle finalement. "Il (le dieu) luttait avec moi en soufflant en moi sa grâce». La métaphore est aisément reconnue par le chœur comme un acte de fécondation. Mais la fille lui échappe, parvient à tromper le dieu : elle évite sa divine grossesse avec les conséquences que l'on connaît : elle parlera, mais n'aura pas le don de persuasion. La possession de Dionysos produit un autre effet. Elle donne plutôt un savoir qui n'est pas le conseil circonstancié d'une stratégie oraculaire mais, sauf quelques cas bien connus, un savoir qui s'offre comme une possibilité diffuse de compréhension globale ${ }^{24}$. C'est

22 PLuT., Sur les oracles de la Pythie, 397c.

23 Sur le pneuma lumineux, cf. R. BULTMANN, Zur Geschichte der Lichtsymbolik im Altertum, in Philologus, 97 (1948), p. 25 sq. L'homologie entre lumière et liquide comme moyen de révélation est clairement attestée dans le Livre IV d'EsDras, pseudépigraphe de l'Ancien Testament du premier siècle ap. J.-C. Cf. A. Pinero-Saenz, Les conceptions de l'inspiration, in C. Kappler (éd.), Apocalypse et voyages dans l'Au-delà, Paris, 1987, p. 157-180. La centralité du processus de l'illumination comme moyen de création et de révélation est bien connue dans le milieu gnostique, cf. G. Filoramo, Il risveglio della gnosi ovvero diventare dio, Bari, 1990 , p. 65.

24 Le caractère oraculaire de Dionysos est bien attesté quoiqu'il n'y ait pas de centre d'une importance comparable aux centres d'Apollon. Mais, même à 
précisément le savoir promis à la cité des Bacchantes. Proches du dieu, les bacchai et les bacchoi, comme le dieu Bacchos lui-même, auront le don de la connaissance dans la folie, ce statut spécial que l'ivresse mythique, devenant politique, peut rendre commun à tous dans un savoir partagé par tous, étrangers et citoyens, jeunes et vieux, hommes et femmes.

Mais la cité des Bacchantes comporte des risques. Dans l'histoire, elle ne fonctionne pas. La pièce d'Euripide le met bien en évidence. Le savoir, la gnosis, de Dionysos Bacchos doit être dépassé par l'impérialisme d'un Dionysos post-politique qui, comme Apollon, possède seulement des intermédiaires spéciaux et rejoint Apollon dans l'engagement de légitimer l'inégalité entre les humains. L'inégalité entre les détenteurs du pouvoir et des humiliés, les génies et les simples, les riches et les pauvres. Dès lors, le message de l'ancien Dionysos se brise dans les mille éclats de l'utopie ${ }^{25}$.

Dipartimento di scienze dell'antichità

Ileana CHIRASSI COLOMBO Università di Trieste

Via dell'Università, 3

I - 34123 TRIESTE

Delphes, une tradition obscure faisait de Dionysos le premier maître du manteion, avant les puissances imparfaitement polythéistes de la Nuit et de Thémis. Cf. Argum. Pind., Pyth. Très intéressant aussi est le rapport lexical établi entre les nymphes du Parnasse, les trois Thries, nourrices d'Apollon, et la situation bachique de l'enthousiasme qui provoque l'oracle : les dérivés indi-

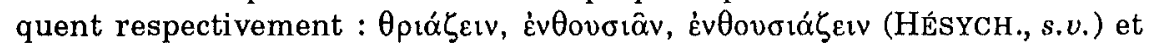
$\theta p ı \hat{\alpha} \sigma \theta \alpha \mathfrak{l}, \mu \alpha v \tau \varepsilon \hat{v} \varepsilon \sigma \theta \alpha \mathfrak{\imath}$ (BEKKER, Anecdota Graeca, 265. Cf. P. Chantraine, Dict. étym. de la langue grecque, Paris, 1983, s.v. $\theta \rho \imath \alpha i$ : $\theta \rho \hat{i} \alpha$. Pour les oracles de Dionysos, cf. Bouché-LeClerCQ, op. cit., p. 373 sq.; cf. aussi P. AMANDRY, op. cit., p. 196 sq.

25 Cf. I. Chirassi Colombo, Dionysos-Bacchos e la città estatica : imagini, messagi e modelli di buon disordine, in Atti del convegno di studi su Dionysos. Mito e Mistero, Comacchio, 3-5 novembre 1989 (sous presse). 